F. DE QUERVAIN

'I'ICHN IS GHE GESTEINSKUNDE 
MINERALOGISGH-GEOTEGHNISGHE REIHE BAND 1

LEHRBÜGHER UND MONOGRAPHIEN AUS DEM GEBIETE DER EXAKTEN WISSENSCHAFTEN 


\title{
Technische Gesteinskunde
}

von

\author{
F. DE QUERVAIN \\ Professor für Technische Petrographie \\ an der Eidgenössischen Technischen Hochschule Zürich
}

Zweite, neubearbeitete Auflage

des gleichnamigen Buches

von A. von Moos und F. DE Quervain

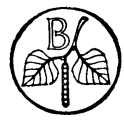

1967

Springer Basel AG 
ISBN 978-3-0348-6882-2 ISBN 978-3-0348-6881-5 (eBook) DOI 10.1007/978-3-0348-6881-5

Nachdruck verboten. Alle Rechte vorbehalten, insbesondere das der Übersetzung in fremde Sprachen und der Reproduktion auf photostatischem Wege oder durch Mikrofilm. Springer Basel AG 1967

(C)

Ursprünglich erschienen bei Birkhäuser Verlag, Basel 1967.

Softcover reprint of the hardcover 2nd edition 1967 


\section{Vorwort}

Das kleine Lehrbuch «Technische Gesteinskunde» gibt vom Standpunkt des Petrographen aus eine Einführung in die Beziehungen zwischen Gestein und wichtigen Gebieten der Technik. Ein Hauptgewicht liegt dabei auf der Darstellung von Eigenschaften der Fest- und Lockergesteine als Vorbereitung auf die den Untergrund berührenden bautechnischen Vorlesungen an Hochschulen oder höhern technischen Lehranstalten und für das Studium der Baugeologie. Ausführlicher ist auch das Gestein als Baustoff und sein Verhalten gegen die Verwitterung im weitesten Sinne behandelt. Mehr als Ergänzung werden die bereits chemische Fragen berührenden Gebiete, wie Gesteine als Rohstoffe der Keramik, der Bindemittel usw., besprochen. Die Lagerstättenpetrographie (Erdöl-, Kohlen- und Erzpetrographie) ist nicht Gegenstand des Buches.

Bei der heutigen Fülle an Darstellungen von Einzelfragen (unter anderem auch in Normenblättern) und an großen handbuchartigen Werken über die technische Seite der Erdwissenschaften war es nicht leicht, eine Auswahl des Wichtigsten und Grundlegendsten zu treffen. Mancher würde etwas andere Gesichtspunkte in den Vordergrund gestellt haben. Im wesentlichen schöpft das Buch aus meiner langjährigen Erfahrung im Unterricht und in gesteinskundlicher Praxis. Die technische Petrographie ist eng mit der technischen Geologie verbunden; sie bildet gewissermaßen deren kleinräumigeren Abschnitt. Die gegenseitige Abgrenzung kann der Natur der Sache nach nicht genau definiert werden, was auch aus der in diesem Buch getroffenen Wahl zum Ausdruck kommt. Vorausgesetzt werden Kenntnisse der allgemeinen Petrographie, wie sie etwa in einer Vorlesung oder in einem Lehrbuch über "Allgemeine Geologie» vermittelt werden.

Um das Verständnis bei den Studierenden der bautechnischen Wissenschaften zu wecken, werden auch einige Begriffe, die über die engere Gesteinskunde hinausgehen, kurz erläutert. Untersuchungsmethoden und Apparaturen konnten im allgemeinen nicht so eingehend beschrieben werden, daß sie direkt anwendbar sind. Dazu muß die Spezialliteratur zu Rate gezogen werden. In der Literaturübersicht wurde versucht, auf die wichtigeren neueren Darstellungen (vor allem in deutscher Sprache) hinzuweisen, wobei auch angrenzende Gebiete einbezogen wurden. Zeitschriftenartikel konnten nur aufgenommen werden, sofern im Text direkt darauf Bezug genommen ist.

Die I 948 erschienene erste Auflage wurde gemeinsam mit meinem Freunde Dr. A. von Moos verfaßt. Zu meinem großen Bedauern war es ihm nicht möglich, sich an der Neubearbeitung zu beteiligen, für die ich somit allein verantwortlich bin. Dies brachte eine gewisse Änderung in der Stoffauswahl mit sich. In manchen Abschnitten, besonders über die Lockergesteine, konnte ich mich indessen weitgehend auf die früheren Ausführungen und die graphischen Darstellungen meines Kollegen stützen. Herrn Prof. Dr. O.W. Flörke, heute in Bochum, verdanke ich wertvolle Beratung auf dem Gebiet der Feuerfeststeine. 
Für mannigfaltige Mithilfe bei der Manuskriptausarbeitung und bei der Korrektur möchte ich Fräulein V.Jenny und Herrn A. STAhel vielmals danken.

Meiner lieben Frau danke ich herzlich für vielfache Unterstützung und für die Zusammenstellung des Registers.

Dem Birkhäuser-Verlag Basel, besonders Herrn C. Ernsele, bin ich zu Dank verpflichtet, daß das Werk in zweiter Auflage in der vorliegenden guten Ausstattung in die Mineralogisch-Geotechnische Reihe der Lehrbücher und Monographien aus dem Gebiet der exakten Wissenschaften aufgenommen werden konnte.

Zürich, Juni i 966

F. DE Quervain 


\section{Inhaltsverzeichnis}

I. Übersicht der wichtigsten gesteinsbildenden Mineralien . . . . . . . . . . . . 11

II. Die Gesteinseigenschaften im Kleinbereich . . . . . . . . . . . . . . . . . 29

1. Die mineralogisch-petrographische Kennzeichnung der Gesteine . . 29

2. Bestimmung des Mengenverhältnisses der Gesteinsgemengteile . . . 32

3. Räumliche Anordnung und Verteilung der Gesteinsmineralien . . 34

a) Lockergesteine . . . . . . . . . . . . . . . . . . . . . . 34

b) Festgesteine . . . . . . . . . . . . . . . . . . . . . 35

4. Korngröße . . . . . . . . . . . . . . . . . . . . . . . . . 38

a) Lockergesteine . . . . . . . . . . . . . . . . . . . . . . 38

b) Festgesteine . . . . . . . . . . . . . . . . . . . . . . . 47

5. Spezifisches Gewicht und Raumgewicht . . . . . . . . . . 49

6. Wasseraufnahme und Porosität . . . . . . . . . . . . . 50

a) Lockergesteine . . . . . . . . . . . . . . . . . . . . . . 53

b) Festgesteine . . . . . . . . . . . . . . . . . . . . . 56

7. Durchlässigkeit und Kapillarität . . . . . . . . . . . . . . . 58

a) Durchlässigkeit für Flüssigkeiten . . . . . . . . . . . . . . 58

b) Durchlässigkeit für Gase . . . . . . . . . . . . . . . . . . 60

c) Kapillare Steighöhe . . . . . . . . . . . . . . . . . . . 60

8. Festigkeit und Konsistenz der Lockergesteine . . . . . . . . . . 61

a) Die Festigkeitsversuche . . . . . . . . . . . . . . . . . . 62

b) Die Konsistenzverhältnisse . . . . . . . . . . . . . . . . . 67

9. Die Festigkeiten der Festgesteine . . . . . . . . . . . . . 71

a) Beziehungen zwischen Festigkeit und Gesteinsaufbau . . . . . 71

b) Die statischen Festigkeiten . . . . . . . . . . . . . . . . . 73

c) Die dynamischen Festigkeiten . . . . . . . . . . . . . . . 74

d) Die Bestimmung der Festigkeiten . . . . . . . . . . . . . . 75

e) Die Elastizitätsverhältnisse . . . . . . . . . . . . . . . . . 78

10. Der Härtebegriff bei Festgesteinen . . . . . . . . . . . . . . . 78

Weitere Prüfungen, die mit der Gesteinshärte zusammenhängen . . 81

11. Weitere physikalische Gesteinseigenschaften . . . . . . . . . . . 81

12. Beschaffenheit der Bruch- und Abnützungsflächen . . . . . . . . 82

13. Bruch- und Abrollformen, Rundung . . . . . . . . . . . . . 84

III. Einige Gesteinseigenschaften des Großbereiches . . . . . . . . . . . . . . . 89

1. Schichtung. . . . . . . . . . . . . . . . . . . . . . . . 89

2. Klüftung . . . . . . . . . . . . . . . . . . . . . . 90

3. Die Bestimmung von Raumgewicht, Porosität, Wassergehalt und Wasserdurchlässigkeit im Großbereich . . . . . . . . . . . 97

4. Festigkeitsuntersuchungen an Lockergesteinen im Verband . . . . 99

a) Die Zusammendrückungsversuche . . . . . . . . . . . . . 99

b) Die Rammsondierung . . . . . . . . . . . . . . . . . . . . . 101

c) Der Tragfähigkeitskoeffizient (Stempelversuch) . . . . . . . . 104

d) Der Scherversuch im Verband . . . . . . . . . . . . . . . 104

5. Die Festigkeitsverhältnisse des Felses . . . . . . . . . . . 106 
6. Die elektrische Leitfähigkeit der Gesteine . . . . . . . . . . . . 107

7. Die Erkundung des Untergrundes und die Probenahme . . . . . 108

a) Die Sondierungs- und Schürfverfahren. . . . . . . . . . . . 108

b) Entnahme von Gesteinsproben . . . . . . . . . . . . . . . 111

8. Die Darstellung der Gesteine in Karten und Profilen . . . . . . . 114

IV. Die Hauptgesteine und ihr wichtigstes technisches Verhalten . . . . . . . . . . 117

A. Die Lockergesteine . . . . . . . . . . . . . . . . . . . . . . . 117

1. Die Lagerung der Lockergesteine . . . . . . . . . . . . . . . 117

2. Die nichtbindigen grob- bis mittelkörnigen Lockergesteine . . . . 119

3. Nicht- bis schwachbindige feinkörnige Lockergesteine . . . . . . 122

4. Die bindigen oder kohärenten fein- oder wechselnd körnigen Lockergesteine . . . . . . . . . . . . . . . . . . . . . . 124

5. Lockergesteine biochemischer Entstehung. . . . . . . . . . . . 126

6. Lockergesteine mit organischen Bestandteilen . . . . . . . . . . 128

7. Die Klassifikation der Lockergesteine nach dem «Unified Soil Classification System» (USCS) . . . . . . . . . . . . . . . . . . 131

8. Die halbfesten Gesteine . . . . . . . . . . . . . . . 132

B. Die Festgesteine . . . . . . . . . . . . . . . . . . . . . . . . 133

1. Die Kalksteine und Dolomitsteine (Karbonatgesteine) . . . . . . 133

a) Die Lagerung der Kalksteine. . . . . . . . . . . . . . . . 136

b) Besondere Erscheinungen an Kalksteinen . . . . . . . . . . 137

c) Technisches Verhalten der Kalksteine und Dolomitgesteine. . . 138

2. Sulfat- und Chloridgesteine (Salzgesteine) . . . . . . . . . . . 146

Technisches Verhalten. . . . . . . . . . . . . . . 146

3. Sandsteine . . . . . . . . . . . . . . . . . . . . . . . . . 148

a) Kennzeichnung . . . . . . . . . . . . . . . . . . . . . . . 148

b) Benennung der Sandsteine . . . . . . . . . . . . . . . . . . . . . 149

c) Die Lagerung der Sandsteine. . . . . . . . . . . . . . . . . 151

d) Technisches Verhalten der Sandsteine . . . . . . . . . . . . 151

4. Die groben Trümmergesteine (Konglomerate, Breccien) . . . . . 153

Eigenschaften und Beständigkeit . . . . . . . . . . . . 154

5. Verschiedene technisch bedeutsame Gesteine . . . . . . . . . . 155

6. Die geschieferten Sedimentgesteine . . . . . . . . . . . . 156

a) Allgemeine Gefügeeigenschaften . . . . . . . . . . . . 156

b) Technisches Verhalten der geschieferten Sedimentgesteine . . 157

7. Die kristallinen Gesteine . . . . . . . . . . . . . . . . . 159

a) Namengebung . . . . . . . . . . . . . . . . . . . . . . . . . . 159

b) Veränderung durch Kornzertrümmerung und Mylonitisierung bei kristallinen Gesteinen . . . . . . . . . . . . . . 160

c) Die Grünsteinausbildung kristalliner Gesteine. . . . . . . . . 163

d) Technisches Verhalten der kristallinen Gesteine. . . . . . . . 165

V. Die Einwirkung der Witterung und der Oberflächenwässer auf Gestein und steinartige Baustoffe . . . . . . . . . . . . . . . . . . . . . . . . . . . . .

A. Die Veränderungen der Gesteine am Naturvorkommen .. . . . . . 177

B. Die Verwitterung steinartiger Baustoffe . . . . . . . . . . . . . . 180

1. Einwirkungen und Reaktionen, die bei der Verwitterung steinartiger Baustoffe in Betracht kommen . . . . . . . . . . . . 180

a) Ohne Mitwirkung von Feuchtigkeit . . . . . . . . . . . . . 181 
b) Mit Beteiligung von Wasser in irgend einem Aggregatzustand. . 182

2. Die Formen der Gesteinsverwitterung . . . . . . . . . . . . . 191

3. Die Bereiche an Bauwerken, die besonders der Verwitterung ausgesetzt sind . . . . . . . . . . . . . . . . . 194

4. Steinschutzmittel . . . . . . . . . . . . . . . 196

C. Der Bodenfrost. . . . . . . . . . . . . . . . . . . . . . 197

VI. Gewinnung und Abtrag von Gestein . . . . . . . . . . . . . . . . . . . 201

A. Die Gewinnung und Verarbeitung von Nutzgestein . . . . . . . . . 201

1. Die Gewinnung von Festgestein . . . . . . . . . . . . . . 201

2. Gewinnung von Lockergestein . . . . . . . . . . . . . . . . 204

3. Die Bearbeitung der Gesteine für Bauzwecke . . . . . . . . . . 206

a) Bausteine . . . . . . . . . . . . . . . . . . . . . . . . 206

b) Gestein für den Straßenbau . . . . . . . . . . . . . . . . 208

B. Der Abtrag von Locker- und Festgestein. . . . . . . . . . . . . . 209

C. Die Silikose . . . . . . . . . . . . . . . . . . . . . . 214

VII. Anforderungen an Gestein nach Anwendungsarten . . . . . . . . . . . . . . 217

A. Gestein als Schüttungsstoff und als verbesserter Baugrund . . . . . . 217

1. Gestein als Schüttungsmaterial . . . . . . . . . . . . . 217

2. Injizierter Baugrund. . . . . . . . . . . . . . . . . . . . . 218

3. Anwendungen von Bentonit im Tiefbau und in der Bohrtechnik . . 220

4. Die Bodenstabilisierung . . . . . . . . . . . . . . . . 221

5. Filtermaterialien im Grundbau . . . . . . . . . . . . . . . 221

B. Zuschlagstoffe für Beton und Mörtel . . . . . . . . . . . . . . . 222

C. Gesteinsmaterial für Straßendecken und Geleisebettung . . . . . . . 225

D. Anforderungen an Bausteine im engeren Sinne . . . . . . . . . . . 228

E. Rohgestein für mineralische Bindemittel . . . . . . . . . . . . . . 230

F. Keramische und verwandte Produkte . . . . . . . . . . . . . . . 233

1. Allgemeines . . . . . . . . . . . . . . . . . . . . . . . 233

2. Baukeramik . . . . . . . . . . . . . . . . . . 236

3. Weitere keramische Produkte . . . . . . . . . . . . . . 237

4. Gesteine als feuerfeste Baustoffe . . . . . . . . . . . . . . . . 238

G. Formsande . . . . . . . . . . . . . . . . . . . . . . . . . . 243

H. Gesteine für verschiedene Spezialzwecke . . . . . . . . . . . . . . 245

1. Leichtbau- und Isoliermaterialien . . . . . . . . . . . . . . . 245

2. Gesteine für Mahl-, Schleif- und Schneidezwecke . . . . . . . . 246

3. Filtermaterialien zur Wasserreinigung . . . . . . . . . . . . . 247

4. Gesteinsmaterial für die Glasfabrikation . . . . . . . . . . . . 248

5. Verschiedene Anwendungen in der chemischen und Hüttenindustrie 249

6. Gesteine für Düngzwecke . . . . . . . . . . . . . . . . . 250

Literatur: Auswahl von Übersichtsdarstellungen, Lehr- und Handbüchern in technischer Gesteinskunde und damit verbundener Wissensgebiete

Sachregister . . . . . . . . . . . . . . . 257 